\title{
Application of Big Data Technology in E-commerce
}

\author{
Zeming Ren \\ Guizhou University of Commerce 550014, Guizhou, China
}

Keywords: Big Data, E-Commerce, Data Mining, User Profile.

\begin{abstract}
With the continuous updating of computer software and hardware technology and the rapid development of the Internet, information technology and human production and life are converging and merging, and data is showing explosive growth. Big data technology for acquiring, processing, analyzing and value extracting of massive data has emerged. More and more people are beginning to learn the brand-new concept of big data, and the country has also issued relevant policies to support the development of the big data industry. The commercial value of big data has been gradually enlarged. Nowadays, the application of big data is very extensive. The development of e-commerce in the era of big data is very rapid. Relying on big data technology to correctly process and analyze the data stored in e-commerce platforms has become the key to the rapid development of e-commerce. Mastering data processing and analysis technology is the key to success for e-commerce companies. This article analyzes the development status of big data and e-commerce, the application of big data technology in e-commerce, and the characteristics, difficulties and innovative advantages of e-commerce development in the era of big data.
\end{abstract}

Domestic e-commerce has achieved rapid development in the context of big data, but it also faces a series of problems and challenges. How to efficiently process the large amount of data generated by the e-commerce platform, and use big data technology to analyze the correlation between the data, and to discover the value hidden in the data has become a top priority for the development of e-commerce, which directly affects the next marketing decision of the company. Collecting, processing, and analyzing consumer behavior data, industry data and transaction data, and making decisions that are conducive to enterprises to carry out precision marketing activities, is exactly what Chinese e-commerce is experiencing. The development of e-commerce in China has gone through three periods: First, when the number of users is the basis, it is profitable by charging advertising fees and membership fees. The disadvantage of this period is that for start-ups with low user stickiness, membership fees are charged too early, and advertising will also affect user experience and lead to user loss. Second, when the sales volume is the basis, the competitiveness and visibility will be improved by increasing advertising and preferential treatment. The disadvantage of this period is the lack of user grouping and precise placement. A large amount of capital investment may not get the expected return, and have little effect. Third is to use big data technology to analyze consumer behavior data, transaction data and e-commerce industry data and other data that can affect the conversion rate of products, and accurately recommend products to users with shopping needs, and achieve accurate recommendations. It is the general direction in the rapid development of Chinese e-commerce, and the realization of these methods is inseparable from the support of big data technology.

\section{The definition and development status of big data and e-commerce}

\subsection{The definition of big data and its development status}

The definition of big data given by mainstream international research institutions is: big data is the collections of diverse, high-dimensional, and massive data. The amount of data influenced by dimensional disasters is too huge, resulting in traditional data processing software unable to meet such large-scale data analysis needs. So big data technology must rely on cloud computing computer resources for analysis. The value density of big data is very low, the amount of data is huge, and the 
data types are diverse, requiring high-speed data processing capabilities. The use of big data technology for data analysis is not about mastering how much data, because not all data is useful. The key meaning of big data technology is to be able to process, store and extract data information from a large amount of data for people to analyze, so as to find useful information. We can compare big data to a gold mining machine. To get more gold, we need to improve the machine's ability to identify and separate gold in the sand. Correspondingly, if companies want to obtain more value, it is necessary to improve the data processing capabilities of big data. Big data processing and analysis require frameworks such as MapReduce to distribute work to hundreds of thousands of computers. The big data processing technologies need to efficiently process large-scale and multi-dimensional data within the waiting time, including massively parallel processing (MPP) databases, data mining, distributed file systems, distributed databases, cloud computing platforms, the Internet, and expand the storage system.

The speed of updating computer hardware is very fast, especially the storage hardware capacity is getting larger and larger, which provides convenience for data storage of big data. Besides, because of the improvement of computer software functions and the increasing stability, big data technology has developed by leaps and bounds under the escort of computer technology. Big data companies have also sprung up. There are mainly three types of big data companies in society. Technology-based big data companies are generally IT companies. IT companies are mainly good at the development and application of data processing modules. This is also a platform for big data to process data. One type is company with good ability to innovate. This type of company values imagination very much. They can often find differences in a bunch of data and put forward new ideas. Finally is data-based companies, like Google, Amazon, Ali, Baidu, etc. The data backend of these companies records a large amount of data, covering all aspects of our lives. These three different types of companies are developing different big data technology industries. However, if the three types of companies work together for coordinate development, the development of big data technology will become more and more diverse and create more value.

\subsection{The definition of e-commerce and its development status}

Based on an open Internet environment, the rapid development of e-commerce is that the Internet provides great convenience for buyers and sellers. In an open network environment, consumers and sellers will not meet in various business activities. Buyers can browse products and complete payments on mobile phones and computers without going out, and sellers can also use mobile computers to complete various operations, such as online purchase and listing of products and setting sales prices. E-commerce is divided into different modes, including ABC, B2B, B2C, C2C, B2M, M2C, B2A (ie B2G), C2A (ie C2G), O2O and so forth. The rapid development of the Internet has produced e-commerce. It can be said that without the Internet, there is no e-commerce. Therefore, certain characteristics of the Internet have become the characteristics of e-commerce. For example, low-cost, high efficiency, openness, and global characteristics of the Internet are also characteristics of e-commerce. These characteristics make the value of e-commerce not limited to being a new type of transaction mode. The openness and high efficiency of the Internet provide unparalleled development space for online business activities, and its outstanding advantages are unmatched by traditional business models.

\section{Application of data analysis technology in e-commerce}

\subsection{User data statistics}

User data statistics is to count user behavior data on e-commerce platforms. Behavior data includes a series of behaviors such as clicking on products, browsing products, and collecting products. What we need to do is to describe the industry or population phenomenon based on the massive data that has been counted. Google is a pioneer in statistical analysis of data. As early as 2008, Google has successfully predicted influenza trends in various regions by detecting influenza-related keywords searched by users. The accuracy of the prediction is almost the same as the report of the U.S. 
Centers for Disease Control and Prevention (CDC). But Google is able to make quick predictions at different times of the day, which is incomparable to the CDC. For influenza, time is the most important thing. Early detection and early prevention can avoid large-scale influenza outbreaks. The successful case of Google predicting influenza reflects the advantages of big data technology in statistical analysis of large amounts of data. China also has some relatively mature products based on big data prediction. For example, the Ali index. Alibaba Index is a professional e-commerce platform market data analysis and prediction product, including market forecasts, industry analysis, which covers all the information left by users and companies on the e-commerce platform, including behavioral data, shopping preferences, consumption trends, and sales volume, product category and other data. It can help brands and shops better understand their product audiences, and clarify the competitive relationship, advantages and disadvantages of enterprises in the industry. It is helpful for enterprises to analyze the advantages and disadvantages of products, and clarify product positioning, clarify the audience of products, and consolidate brand influence.

\subsection{Data mining technology}

The process of data mining is the process of knowledge discovery. Reasonable use of data mining algorithms such as attribute screening, clustering, association, regression, etc., to explore the internal connections between commodities, between groups of people, between commodities and groups of people, is equivalent to a pair of bright eyes that can discover marketing entry points in time and help companies explore greater business opportunities. Data mining can solve the problem that users who buy a certain product will generally buy other products, and those who like this thing will also like other things. These are also the problems that e-commerce platforms are facing. How to accurately analyze user preferences, effectively recommend users' favorite products and stimulate user consumption can bring more profits to enterprises. Part of this involves the association analysis algorithm in data mining. When it comes to association analysis, the most classic case is the bundle sale of beer and diapers. Wal-Mart supermarket found that some people would buy beer and diapers after getting off work on Friday, so they promoted beer and diapers together on Friday, and finally achieved satisfactory results. In people's subjective consciousness, beer and diapers seem to have no actual connection, but through analysis based on user data statistics, Wal-Mart Supermarket found that there is a certain connection between these two products, so the combination of these two products brings greater profits to the mall. We usually encounter this situation when browsing products on e-commerce platforms. For example, Taobao's "good product recommendations" and "guess your favorite" are accurate recommendations based on statistics of a large number of user behavior data.

\subsection{User porfile technology}

Users will leave a variety of data while browsing and purchasing products on the e-commerce platform. User profile technology is to put different labels on users based on these data. These labels include users' shopping habits, consumption behaviors, and industries.

Obtain user behavior data can record and classify users into different groups, such as students, teachers, and IT practitioners. Such classification can facilitate enterprises to realize classified marketing. This is the core principle of user profile technology. In the field of e-commerce in China, JD.com and Ali's servers record a large amount of high-quality, multi-dimensional user behavior data. User profiles are obtained by categorizing these large amounts of user behavior data to describe users' shopping preferences, social circles, and shopping capabilities, thus can get different user tag models, and then dig out the huge business opportunities hidden in the massive data. JD.com and Ali mainly apply user portrait technology to search and advertising recommendations. When shopping on JD.com and Taobao, the platform will recommend products related to items you have bought. For example, if we often search for height-enhancing insoles, and we will see recommendations for height-enhancing insoles on the homepage. This is the recommendation mechanism based on the evolution of user profile technology. Advertisements are also accurately placed according to your browsing preferences of the software you usually use, so you will feel that the software understands you more and more. User profile provides technical support for consumers to find their favorite 
products among the dazzling array of products, and provide businesses with a basis for operation and decision-making at the same time.

\subsection{User group analysis}

According to the group of users, different user types can use different marketing methods. For example, if the purchasing power of a certain group of people is not strong, you can stimulate consumption through crowd discounts and issuing coupons. Users can be divided into four different groups according to the latest shopping time, shopping frequency, shopping quantity, purchasing ability and other tags, which are high-value users, high-quality users, ordinary users and low-contribution users. After dividing users into several groups, we can easily analyze and formulate marketing strategies, and use different marketing methods for different user groups. As far as high-value users are concerned, high-value users have a strong sense of dependence and trust in our products, so there is no need for excessive management. Low-contributing users are mainly analyzed. The main reason for the low buying interest of low-contributing users is that the purchasing power of users is not high, or the discount of platform products is too low. If the discount power of the platform is too low, in order to increase the users' interest in shopping, we can use the form of issuing coupons to stimulate consumption. If the users' own purchasing power is insufficient, we can stimulate the users' desire to buy through recommending low-priced goods.

\subsection{Realization of precision marketing}

Precision marketing actually solves the kind of content to send to users at what time. We cannot achieve this without the so called user portraits. The user portrait has labeled all the user categories, and only needs to send the right information to the user at the right time. We usually receive different shopping advertisements, that is, we have been labeled with different labels. Our shopping interests are analyzed through user portraits, and then these are sent to us through advertisements, text messages, emails, etc. Alibaba and JD.com usually add multi-dimensional tags to users based on a series of behaviors such as browsing, clicking, consulting, adding attention, and adding shopping carts, and then use mobile phone text messages, software pop-up reminders, and advertisement push to recommend product information to appropriate users. Of course, any possible situation should be considered. For example, the user's favorite product is out of stock. At this time, the user has a strong desire to buy. After a period of waiting, how can the shop attract users to buy again after the product arrives? The user collects one certain product and puts it into the shopping cart, which shows that the user likes this product, so why don't the user buy it directly? How should we sell in order to make users determined to buy and facilitate users to buy? In order to solve these problems, we can use user portrait technology. According to the user's usual shopping preferences and purchasing power, the user is labeled with the category and price of the user's favorite goods, and the next time the recommendation is to analyze whether the price is sensitive and whether it is reasonable. After the goods arrive, they can be pushed appropriately and accurately according to the user tags. Appropriate push will not only generate clicks and transactions, but also help users save time and get a better shopping experience, thereby increasing goodwill and creating dependence.

\section{The development of e-commerce in the era of big data}

\subsection{E-commerce and its characteristics in the era of big data}

In the context of big data, the effective use of the collection of big data, processing and analysis technology promotes the sustainable development of e-commerce and provides great convenience for better reflecting the value of e-commerce. E-commerce is a series of new forms of economic development and consumption. Like other forms of Internet economy, e-commerce will also generate a lot of data, including e-commerce platforms, mobile terminals, different social platforms or any other third-party platforms. How can we make full use of e-commerce data to provide more effective and valuable information? Traditional data processing methods cannot solve this problem. The amount of data generated by e-commerce is not only huge, but also diverse. The data generated by 
e-commerce also includes user behavior data and various feedback comments on customers' shopping on e-commerce platforms. Accurate analysis of these data can help companies understand user needs, classify user consumption trends, and help companies adjust sales plans and increase sales profits through consumption trends. The application of big data technology in e-commerce has greatly changed the business model of e-commerce, from the traditional offline sales model to the information-based online data analysis model. Through professional data analysis, e-commerce can accurately predict consumption habits and consumer psychology, thereby providing a series of suggestions and guidance for the commercialization of e-commerce, reducing e-commerce decision-making and time costs, and improving efficiency. In addition, the application of big data technology can make industry-specific comparisons of data generated by e-commerce, which improves the convenience of resource sharing. The data resources generated by e-commerce platforms can not only be used by themselves, they can also bring corresponding commercial benefits to enterprises.

\subsection{Difficulties encountered in data resource sharing in the era of big data}

Although big data technology has now developed more maturely, the value discovery of data has not been perfectly demonstrated. The key is that there are relatively few applications in data resource sharing, and there are still many difficulties in sharing comprehensive resource. There are many reasons for this. First, companies and governments are unwilling to spend too much time processing these data. Second, there is no corresponding technology, which consumes too much time and energy, resulting in a backlog of large amounts of data and the value of data cannot be displayed. Although some companies have realized the importance of big data, the use of data is still very limited due to their own technical limitations. In addition, companies with big data processing capabilities have certain limitations in data sharing. They know that this can bring benefits to the society and better development for the enterprise, but data is the foundation of a company. If data resource sharing is realized, it will inevitably lead to the leakage of important data. Although it is profitable, in comparison, data blockade is preferred to resource sharing. Such behavior actually hinders the development of e-commerce. Of course, there is another reason. Although there is a large amount of data, the useful information is poor. With technical limitations, data processing is not efficient. The rapid growth of information makes information processing more difficult, which requires the development of big data technology to keep pace with data growth.

\subsection{E-commerce service model innovation in the era of big data}

Big data has injected new vitality into the production and development of enterprises and brought great convenience to human life. The application of big data technology in e-commerce is even more powerful. Different people have different consumption views, some like high-quality products with low-cost, and some pursue products that can meet their own taste and improve the quality of life. Different shopping habits bring different shopping data. The more data, the better the analysis, and the upgrade of e-commerce services is inseparable from the results of these data analysis. The classification of user consumption habits of e-commerce platforms is inseparable from data resources. Through the processing and analysis of user consumption data, different users can be provided with different services. Users who pursue cost-effectiveness can buy products with the ultimate cost-effectiveness, and users who pursue quality of life can buy high-quality goods. Satisfying the different consumer desires of users can improve users' shopping experience, and promote consumption to the greatest extent. In the era of big data, e-commerce companies need to use the information resources and analysis results generated by big data to upgrade and subdivide e-commerce service models, analyze their own advantages and disadvantages, and formulate the best route for their own development. Many large e-commerce platforms are unparalleled in scale and product categories. In this case, if you want to seek development, you must start from the details and create your own service goals in the specific e-commerce field. Only by providing high-quality e-commerce services can we have the opportunity to stand out from many e-commerce companies. Big data combined with the innovative model of e-commerce has opened up new ideas for the development of e-commerce enterprise services. Traditional e-commerce service innovation always 
has many limitations. However, information analysis under the background of big data has created a brand-new e-commerce service model. Based on user consumption data analysis and application, the e-commerce platform can bring guiding opinions for all aspects of enterprise services to develop personalized e-commerce service models that are more suitable for consumer groups. This greatly reduces the pressure on enterprises, shortens the decision-making time, and helps enterprises develop a correct and rapid development route at the first time. Today, consumption patterns have gradually changed from traditional tangible consumption to multiple consumption patterns that combine tangible and e-commerce. Collecting sales data from various channels, analyzing and understanding it will bring guidance to the innovation and development of e-commerce, thus facilitating different shopping needs of consumers, and injecting new vitality into social and economic development. The status of e-commerce in the socio-economic field has become increasingly important, which makes cloud computing and big data begin to become the core of enterprise development. The use of its technology also provides a better direction and way for the innovation of e-commerce service models. The application of big data in the field of e-commerce services can not only lead the development direction of enterprises, but also create new business models in various fields of social and economic development.

\section{Conclusion}

This article introduces the big data technologies used in e-commerce, such as attribute screening, clustering, correlation, and regression. The rational use of big data technology by e-commerce companies can inject new vitality into the development of enterprises and provide new creative suggestions for the upgrading of business service models. The development of big data technology not only brings new creative space to e-commerce enterprises, but also brings considerable market value. Companies need to understand e-commerce big data to accurately understand consumer needs. The innovation of e-commerce service model in the era of big data will not only not cause the loss of old customers, but also attract the attention of many new customers. This can not only reduce the cost of enterprise investment, but also increase the profit of the enterprise to a large extent. Moreover, big data technology can also guide the development direction of e-commerce, allowing enterprises to seize the opportunity to avoid business losses due to hindsight or wrong decisions.

\section{References}

[1] Huang Xindan, Lin Zikun, Jiang Xinmiao. Application of big data marketing in e-commerce [J]. Consumer Guide, 2015, 99.

[2] Sun Jingnan. On the promotion of big data analysis in e-commerce [J]. China Management Informationization, 2016.

[3] Wang Jiwei. On the application of big data marketing in e-commerce [J]. Journal of Hubei Correspondence University, 2015

[4] Zhang Xiaonuo. Using big data technology to analyze customer loyalty in e-commerce [J]. China Science and Technology Information, 2015.

[5] Zhang Qinghe. Analysis of e-commerce marketing management under the background of big data [J]. Manager, 2016. 